\title{
Infrared Thermal Wave Nondestructive Testing Technology and Its Application
}

\author{
Yang Zhou ${ }^{a}$, Jing Cai ${ }^{b}$ \\ Changcheng Institute of Metrology \& Measurement, Beijing 100019, China \\ azhousally1117@163.com, bcaijing@cimm.com.cn
}

\begin{abstract}
The technical principles and characteristics of the infrared thermal wave nondestructive testing technology have been reviewed in this paper. Compared with five conventional nondestructive testing methods, the advantages of infrared thermal wave nondestructive detection technology are highlighted. In addition, this paper describes the applications of infrared thermal wave nondestructive testing technology in some fields that conventional nondestructive testing techniques are difficult to solve, including composites, honeycomb structures, wind turbine blades, etc. The technical features and wide application advantages of infrared thermal wave nondestructive testing have been presented in detail.
\end{abstract}

Keywords: Thermal wave; Nondestructive testing; Composites; Honeycomb structure; Wind turbine blades.

\section{Introduction}

Infrared thermal wave nondestructive testing technology is a new-developed nondestructive testing technology. Further studies towards the application of infrared thermal wave nondestructive testing technology is important to the improvement of the safety and reliability in aerospace vehicles, industrial and civil devices. Infrared thermal wave nondestructive detection technology is different from the other five conventional nondestructive testing technology, which is shown non-contact, fast detection speed, and small curvature influence advantages. Furthermore, it can solve some problems that conventional nondestructive testing technology cannot. So it has become a new and promising nondestructive testing technology.

\section{Conventional nondestructive testing technology}

\subsection{Ultrasonic testing}

Generally the frequency in the range of $25 \mathrm{MHz} \sim 2 \mathrm{KHz}$ of the sound wave is called ultrasonic. It is a kind of mechanical wave, generated by the mechanical vibration source shock excitation in elastic medium, and the essence is permeated to the interior of the object through the form of stress wave and spread. Ultrasound has a variety of propagation characteristics, such as reflection and refraction, scattering and diffraction, resonance and attenuation, etc, which could be utilized in of the detection. When the ultrasonic wave spreads in the interface of two different acoustic impedance mediums, it will be reflected. The energy reflected back is related to the orientation and the size of interface, as well as the difference of the acoustic impedance of the two sides. So it can be used to detect the internal defect through the energy of the reflected wave. Ultrasonic testing is suitable for the detection of internal area type objects, but it is difficult for the detection of small, thin and complex parts. Furthermore, the ultrasonic testing needs coupling agent for the coupling, and serious scattering happened when detect coarse-grained materials, which would make it difficult to detect accurately. The ultrasonic testing is also very slow and needs a long testing period.

\section{2 $\mathrm{X}$ - ray testing}

When $\mathrm{X}$ ray through the detected object, the defective parts for ray absorption capacity is higher than intact parts. Therefore, it can be detected by detecting the differences in the ray intensity to estimate whether there is a defect in the test object. This method is not limited by the material and geometric shape, and it can maintain a permanent record, notably, X-ray testing is very sensitive for 
the detection of volume type defects such as porosity, falling off, and slag. But the investment of X ray equipment is very large and the vertical direction of the crack is not easy to be found. Moreover, the installation and safety aspects have strict requirements, and it is not suitable for on-site monitoring, long monitoring period is also required.

\subsection{Magnetic particle testing}

Detection of magnetized internal magnetic flux, as there is discontinuous and local distortion, which leads to a portion of the magnetic field never continuous emission, and this phenomenon is called the magnetic flux leakage. The leakage flux has the ability to adsorb the magnetic material, thus forming the characterization of the corresponding with the discontinuous profile. The surface defects of ferromagnetic materials are detected by magnetic particle detection method, which has a higher sensitivity than ultrasonic or radiographic ones, and it is easy to operate, the results are reliable and visualized. But this method is limited to ferromagnetic materials, and it is difficult to determine the depth of defects quantitatively. Besides it is not suitable for the detection of austenitic steel, non-ferrous metals, non-metallic materials and the insulting materials.

\subsection{Penetration testing}

Penetration testing is used to detect the defects through the detection of liquid surface tension, which is related to the wetting and emulsification effect. The testing agent is smeared to the surface of the inspected object, then it will penetrate into the defect. Removal of excess agent on the surface, and then coated with a developer, the traces of defect image can be observed, so the test piece defects can be examined by naked eyes. This method is simple, economic, and highly reliable to detect the crack, surface defects such as cracks, porosity. Its special advantages can be shown in the detection of large, irregular parts and local parts. But the process of penetration testing is complex, and the testing agent is easy to volatile, and it can be used to detect the surface-opening defects only.

\subsection{Eddy current testing}

If the coil is connected to the alternating current, the current passing through under certain conditions is unchanged. If the coil is close to the measured workpiece, like the boat in the water, the eddy current will be induced within the workpiece. Influenced by the eddy current, the coil current will change [1]. The size, phase and flow pattern are affected by the performance and defect of the test piece, and the eddy current reacts to the magnetic field, which will cause the change of the coil impedance. So the changes of the coil current can reflect the workpiece has defects or not. Eddy current testing has very high detection sensitivity to the metal surface and near surface defects, but it is not practicable for the detection of nonmetal coated with thermal barrier.

\section{Thermal wave nondestructive testing technique}

Infrared thermal wave non-destructive detection technology is a kind of new non-destructive technology, which takes advantage of the interaction between variable heat source (thermal excitation) and the structures of media materials and media to detect the nonuniformity or abnormal situations (damages and defects). For the variable temperature field (thermal wave) applied to the object, the physical properties and boundary conditions of the surface of media materials and under the surface will influence the transmission of heat in a special form and the changes of thermal field on the surface of media surface in one form, namely reflecting it on heat map. The uniformity information of materials and the structure information below its surface can be acquired by controlling thermal excitation method and measuring the thermal field changes on the surface of materials.

If there is a defect in the sample, there will be a difference in thermal conductivity between the defect and the normal part. After a certain time, these defect positions of the samples will result heat increasing or loss and cause the change of the surface temperature gradient. Therefore, the infrared temperature measuring instruments can be used to scan and measure the temperature distribution of the sample surface. When the temperature mutates, it means that these positions of the surface or the inner exist defects. 
Infrared thermal wave nondestructive detection technology designs the heat sources (flashlight, laser, ultrasonic wave, $\mathrm{THz}$ wave, hot wind, electromagnetism) with different features according to the materials, structure and type of defects of tested object and thermally excites the tested object in the forms of period, pulse, ladder and other function forms under the controlling of computer and special software, collects the data about time sequence thermal wave signals with infrared thermal imager and uses special software to process data, and finally shows the test results in the form of image to reach the purpose of detect damages and defects characterization. Taking the test piece with inner lamination defects as an example, its sketch map of operating principle is shown in the following figure1.

(1) High-energy flashlight instantaneously produces plane heat sources to heat the surface of tested object;

(2)Thermal waves transmit inside test pieces and are blocked at the place with thermal insulation defects;

(3) Heats accumulate on the surface of test pieces with lamination defects causing its temperature differences with other areas;

(4) Infrared thermal imager is used to record the infrared radiation on the surface of materials;

(5) Computer processes the collected data and shows images.

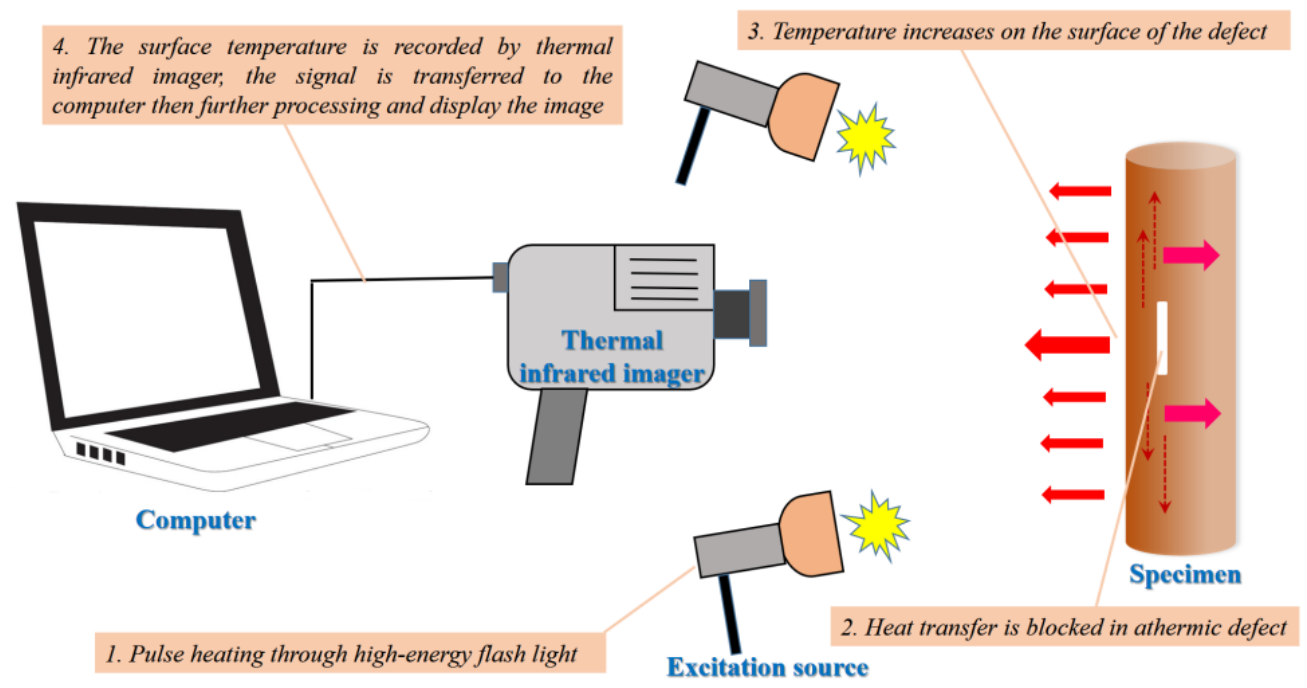

Fig1 Schematic principle of nondestructive testing of high energy flash heat excitation system The features of infrared thermal wave nondestructive detection technology:

a) No contact, high speed, and no contamination of or contact with test piece;

b) Visual judgment of heat map. The position and size of defects can be found directly;

c) For the testing of parts or products with any shape or structure, the test results don't have too many influences on curved surface;

d) Lightness and moveable probe. Suitable for field use;

e) Wide application fields. Almost all metals and nonmetals can be detected with the method;

f) Wide measurement area. Single measurement can cover square meter magnitude.

\section{Application case}

\subsection{Turbine blade thermal barrier coating detection}

Turbine blade is a key hot end component of turbine engine, which works at the high temperature environment. In order to provide thermal protection of turbine blade, reduce blade basal body temperature, prolong the service life, usually the high temperature parts of turbine blade is coated with the thermal barrier coatings (TBC).[2] The basic principle of TBC is to make use of ceramic material which is high temperature resistant, corrosion resistance, good performance of wear resistance and 
good heat insulation performance features, it can be precipitated to the alloy surface through the coating deposition method, so as to realize adiabatic, reduce the surface temperature, improving the thermal efficiency of the engine. However, the thermal barrier coating is composed of basal, bonding layer and ceramic layer. Each level has different physical, thermal and mechanical properties. Complex structures and harsh working environment make thermal barrier coating may have defects in the preparation and use, and a defect will directly influence the quality of the turbine blades.

In ultrasonic testing, ultrasonic will spread to internal parts through coupling agent, the distance between the defect and surface can be determined by measuring the round-trip time of the signals. This method utilizes the object deformation or force waves of cracks to test information of inner crack directly, it is one of the most widely 0e1nough echo, so ultrasonic testing is not suitable for the nondestructive testing of TBC. Penetration detection utilizes the yellow green fluorescent penetrating liquid or red dye penetration liquid, they are easy to permeate into the narrow gaps, so the non-contact detection cannot be achieved. Eddy detection utilizes electromagnetic induction effect, different metal materials show different phases and amplitudes in alternating current. Because TBC is non-metallic material, so the eddy detection cannot be applied in the detection of TBC. Similar to eddy detection, the magnetic particle method is suitable for the magnetic metal material only, non-metallic and insulating materials should not be tested using this method. The X-ray testing has a low sensitivity in the detection of layered defects. Conventional methods have low sensitivity, complex operation, and show different limitations, which cannot be applied in the non-destructive testing of TBC materials.

Infrared thermal wave NDT technology has shown non-contact, high detection rate, minor effect of the curvature advantages, which make it ideal for TBC defect detection.

When the turbine blade coating is damaged, the firing rate have obvious difference between damaged and intact parts. A large number of experiments have shown that the emissivity of the thermal barrier coating part is nearly 0.95 , while the uncoated part is around 0.55. [3]

On the other hand, the different emissivity will have significant differences on the radiation intensity at the same temperature. When the temperature increases, temperature difference will also increase. When the temperature of blade reaches $1000 \mathrm{~K}$, the radiation intensity of the intact parts will be $72 \%$ higher than the damaged parts. Different emissivity produces different radiant intensity and it will show obvious differences in infrared imagery, thus we can easily judge the defect part.

When the temperature is heated to a certain degree, then cooled naturally, the heat exchange between the damaged parts and air will accelerate, temperature decreases will also accelerate, so in the infrared image it will show obvious temperature difference with the intact parts.

In conclusion, the intact parts are coated with thermal barrier, which belong to non-metallic, and the damaged parts are equal to exposed metal parts, the emission rate of them are very different. Therefore, in the same temperature, the dark region of the infrared image represents the damaged area. The infrared image of the turbine blade is shown in Figure 2. [1]

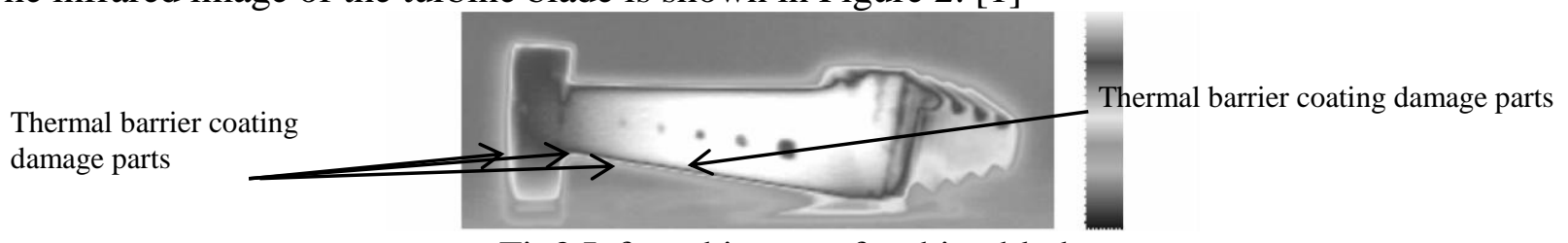

Fig2 Infrared image of turbine blade

\subsection{Detection of honeycomb structure}

The honeycomb structure has the advantages of high strength, light weight, high rigidity and so on. It is a kind of structure which is often used in the field of aerospace, shipbuilding and other fields. In the process of making and using, the honeycomb structure is prone to water and debonding, so it is necessary to carry out nondestructive testing.

Honeycomb structure is an object which is difficult to be detected by conventional detection methods. The general test hole, honeycomb structure using $\mathrm{X}$ radiographic testing method of osteoporosis, fracture, joint detachment from honeycomb joints and other defects. But because the 
skin is thin, the conventional method cannot accurately detect the defects, and infrared thermal wave technique for the detection of intuitive judgment, accurate detection, so it is very suitable for all kinds of flaws of the honeycomb structure detection. [4] When the honeycomb nodes separate, debonding part will be exposed in the air. Because of the low thermal conductivity of air, it will lead to temperature difference on the structure surface. The defective surface forms a low temperature area in the thermal image, which can be judged and detected the defects. Cellular body specimen (bare surface) physical diagram is shown in figure 3, Aluminum honeycomb sample infrared thermal imaging test results as shown in Figure 4. [5]

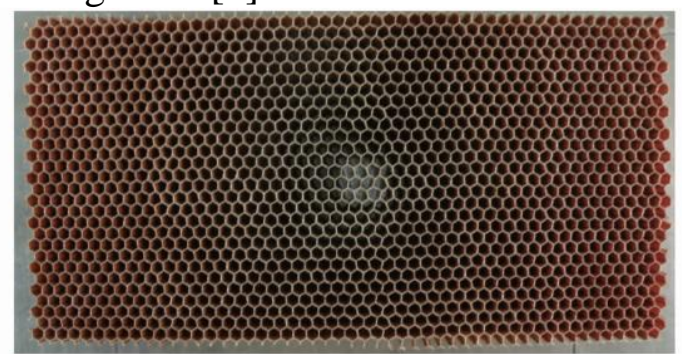

Fig.3The physical map of the cellular mechanism (naked nucleus)

Debonding zone

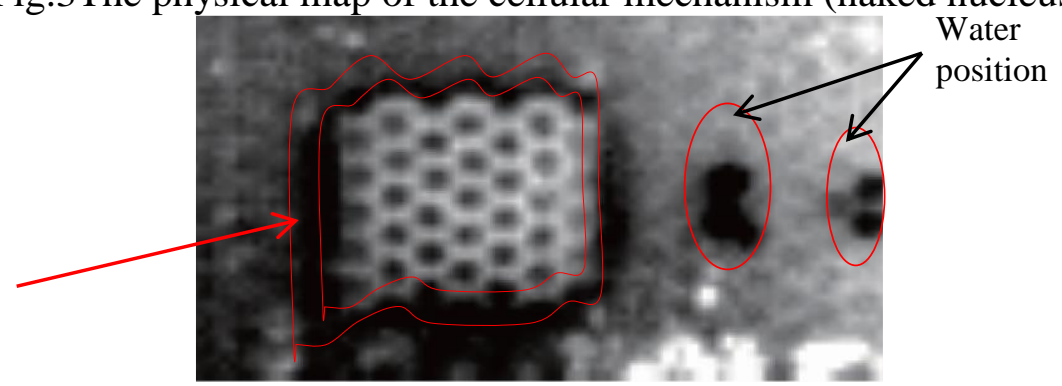

Fig4. Aluminium honeycomb samples of infrared thermal imaging detection results

\subsection{The detection of wind-power blades}

Wind turbine blade is one of the most critical components of the wind turbine. With the improvement of single wind generator power, the wind turbine blade is becoming more and larger. With the improvement of the material and technology level, the quality performance requirements are also getting higher and higher. However, there are still no more mature detection methods used in wind power blades in China. Internationally, the infrared, ultrasonic, ray detection methods have been tried in the detection of wind turbine blades, but all of them are still in the laboratory research stage. In China, the quality control is carried out mainly through the static test, shape inspection, visual inspection, knock and so on. [6]

Gel coat is prone to damaged and form the blisters in the production of wind turbine blade. When the wind motors run, the blisters can increase the resistance, the speed will reduce, and easy to generate seeper. Especially in the rainy season, if the water accumulation in the blisters, the lightning protection index will be greatly reduced. Using the infrared thermal wave nondestructive testing technology to test blisters on the surface of the blade, which can provide certain reference basis for the quality control of blade. The leaves near the surface of thermal infrared detection for blisters as shown in Figure5. [7]

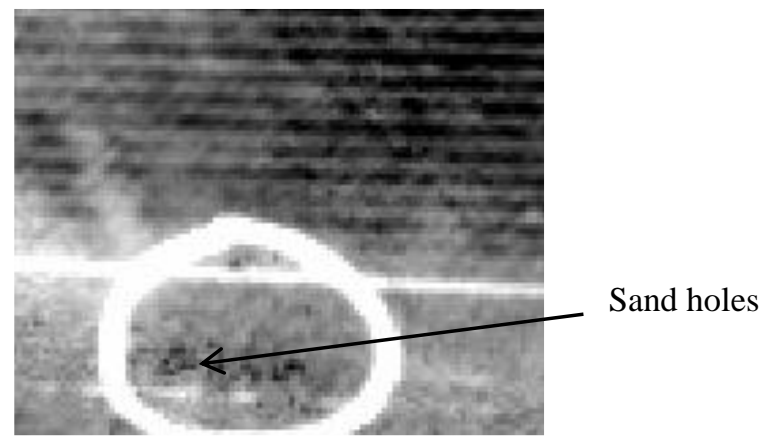

Fig5 The thermograpgic image of air pockets defect in turbine blade 
In the infusion process of wind turbine blade, the resin system would introduce the bubble, the limitations of perfusion process will lead to local fiber infiltration isn't saturated completely, the bubble in the process of forming the blade is not exhausted completely, the core material diversion effect is not ideal, and easy to form the white spot defects. White spot area has a larger thermal resistance than normal area, it will prevent the heat transmission from the surface into depth, so the white spot area of the surface has a higher temperature, which shows a higher brightness in the heat map. As shown in figure 6 a heat map for white spot flaw detection. [7]

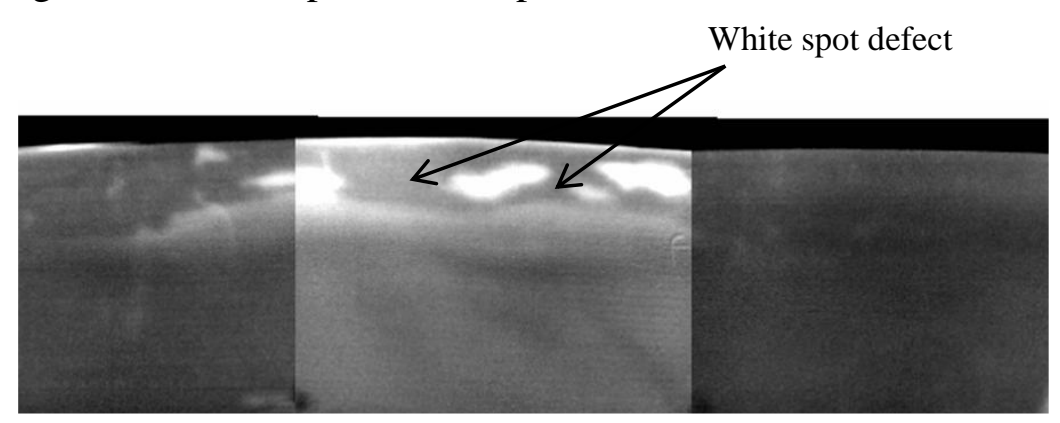

Fig6 The thermographic image of white spot defect in turbine blade

\section{Summary}

Infrared thermal wave nondestructive testing technique has the advantages of non-contact, intuitive, large detection area, can be a powerful complement of conventional detection methods. With the rapid development of advanced composite in aviation, spaceflight, widely used for chemical industry, ship. Infrared thermal wave nondestructive testing technology in ensuring the safety, quality control, and prolong service life, etc, play an important role. With the rapid development of advanced composite materials and its in the aviation, aerospace, chemical, shipbuilding, etc, are widely used, the infrared thermal wave nondestructive testing technology in the protection of its safety performance, quality control, and prolong the service life of the plays an important role. In addition, the infrared thermal wave nondestructive testing technique is introduced into the field of wind power blade, which provides a new direction of non-destructive testing of wind turbine blades. Infrared thermal wave nondestructive detection technology for quality control in the production and use of wind turbine blades, and provide an important basis for the future of wind turbine blades to quality control and technical improvement.

\section{References}

[1]. FENG Chi, HUA Xiang, Applications of the infrared thermal wave technology in thermal barrier coating testing, Applied Science and Technology.Vol.42 No.1, (2015), p.16.

[2]. LIU Zhan-wei, ZHU Wen-ying, SHI Wen-xiong, et al. Thermal barrier coating nondestructive testing technology progress, Aviation manufacturing technology. 4 (2016) 43-44.

[3]. MARKHAM J R, LATVAKOSKI H M, FRANK S L F, et al. Simultaneous short and long wavelength infrared pyrometer measurement in a heavy-duty gas turbine [J]. Journal of Engineering for Gas Turbines and Power, 2002, 124 (3):528-533.

[4]. LI Yan-hong, JIN Wan-ping, YANG Dang-gang, et al. Thermal wave nondestructive testing of honeycomb structure, Infrared and Laser Engineering. Vol.35, No. 1 (2006) 45-46.

[5]. HUANG Xin-ping, TAO Ning, JIANG Yu-long, et al. Thermal Wave Nondestructive Testing of Honeycomb Defects and Finite Element Modeling, ACTA OPTICA SINICA.Vol.33,No.6 (2013) 0612002-5. 
[6]. XIAO Yan-song, YAN Tian-ming, Wind turbine blade numerical studies of infrared thermal imaging nondestructive testing technology [J]. Journal of Beijing university of technology, 2006, $32(1): 48-52$.

[7]. YUE Da-hao, LI Xiao-li, ZHANG Hao-jun, et al.IR Thermography Inspection of Wind Turbine Blades, Infrared Technology.Vol.33 No.10, (2011) 615-616. 